Reducing the use of high dose antipsychotic medication in acute adult inpatient psychiatric units

Shay-Anne Pantall ${ }^{1 *}$, Sarah Warwicker ${ }^{2}$ and Lisa Brownell ${ }^{1}$

${ }^{1}$ Birmingham and Solihull Mental Health NHS Foundation Trust and

${ }^{2}$ University of Birmingham

${ }^{\star}$ Corresponding author.

doi: 10.1192/bjo.2021.893

Aims. To evaluate the use of antipsychotics, and high dose antipsychotic treatment (HDAT) in psychiatric inpatient units

Background. The Royal College of Psychiatrists published a consensus statement on high dose antipsychotic medication in October 1993. Such treatment carries an increased risk of adverse effects including towards ventricular tachycardia and sudden death. Method. A retrospective case note review of all male patients on acute adult inpatient units in a psychiatric hospital in South Birmingham on a date in June $2018(\mathrm{n}=45)$ including review of electronic patient records and prescriptions. This was compared with the results of an earlier study, with identical methods, undertaken in June 2015.

Result.

- In both 2015 and 2018, only a minority of patients $(20 \%$ and $11 \%$ respectively) were informal.

- In both 2015 and 2018, the majority of inpatients had a diagnosis of schizophrenia (54\% and $67 \%$ )

- In both 2015 and 2018, 93\% inpatients were prescribed antipsychotic medication.

- In 2015, 56\% patients were prescribed HDAT. This reduced in 2018 to $16 \%$.

- This reduction in use of HDAT was almost entirely due to a reduction in the prescription of PRN antipsychotic medication.

- In terms of regularly prescribed antipsychotic medication, in both years, the most commonly prescribed drug was flupentixol, with a range of other second generation oral and long acting medications being prescribed, usually at doses within BNF limits.

Between the two years, there was a substantial change in the prescribing of PRN antipsychotics. In 2015, 59\% individuals were prescribed at least one PRN antipsychotic (27\% were prescribed two). In 2018 , this reduced to $40 \%$ prescribed at least one, and only $2 \%$ being prescribed 2 PRN antipsychotics. In both years, oral quetiapine was a common choice (39\% patients in 2015 prescribed oral quetiapine, and $34 \%$ in 2018). In 2015, 39\% patients were prescribed oral or intramuscular aripiprazole, while this reduced to 7\% in 2018 . Conclusion. The vast majority of psychiatric inpatients were being prescribed antipsychotic medication. Prescription of high dose antipsychotic medication was common in 2015, and this was largely attributable to high levels of prescribing of PRN antipsychotics. Following an educational programme for junior doctors and ward nurses, and the introduction of electronic prescribing, we achieved a significant change in practice, particularly in the prescribing of PRN antipsychotics, which has reduced our patients' risk of receiving high dose antipsychotic medication.

Audit: children \& young peoples's services depression pathway Tees, Esk \& Wear Valley Trust wide compliance

Mary Parker ${ }^{\star}$ and Veenu Gupta

Tees, Esk and Wear Valleys NHS Foundation Trust

${ }^{\star}$ Corresponding author.

doi: 10.1192/bjo.2021.894
Background. This complete cycle consists of an audit assessing compliance with the evidence based person-centred pathway of care for Depression in Children and Young People across the Trust, completion of an action plan and re-audit of progress, aiming to improve patient care. The pathway was derived from NICE Quality Standard 48/Clinical Guideline 28, updated for NICE Guideline 134 (2019) and includes comprehensive assessment considering comorbidities, social, educational and family context, parent/carer Mental Health assessment and plan for treatment including psychological therapies as first line treatment in mild depression.

Method. The Audit tool was compiled from the above evidence based pathway and NICE guidance. Each of the 26 community teams were requested to select 5 cases on the pathway who had completed a minimum of 6 treatment sessions (final sample size $\mathrm{n}=61$ ). The results were analysed for compliance against the pathway and compared with previous results by the clinical audit team.

Result. The results showed areas of good practice, maintained and improved on re-audit, with over $90 \%$ compliance in key evidence based areas regarding consideration of comorbidity, social and educational context and $100 \%$ compliance in offering psychological interventions.

Improvement was obtained in some areas highlighted in the previous audit e.g. poor recording of ICD 10 diagnosis in medical records, $19 \%$, improved to $30 \%$, and less than $40 \%$ recording of symptom tracking via the RCADS (Revised Children's Anxiety and Depression Scale) monitoring improved to over 50\%. There had been a failure to record identification or referral to other pathways/services for mild depression in the 16-18 age group with $0 \%$ compliance; this improved to $82 \%$ and $100 \%$ respectively.

Areas still needing improvement were highlighted including recording of weekly monitoring of medication side-effects for first 4 weeks (43\%) and a referral of parent/carers to mental health services after identifying issues (40\%).

Response to the audit also improved significantly from $29 \%$ of teams not responding in the initial audit to a limitation of only 1 of $26(4 \%)$ at re-audit.

Conclusion. This audit cycle has demonstrated that use of an evidence based approach has been instrumental in improving patient care. The Audit evidenced areas of good practice in holistic assessment and use of psychological therapies and importantly highlighted areas of significant improvement needed including initial monitoring of medication response and referral onwards of parents/carers with mental health issues. Continuous improvement in patient care is planned via a targeted action plan, and further re-audit.

\section{Changes in patients characteristics and service provision in liaison psychiatry during the COVID-19 pandemic}

Frederica Passos ${ }^{1 \star}$, Miguel Constante ${ }^{1}$, André Delgado ${ }^{1}$ and Maria João Heitor ${ }^{2}$

${ }^{1}$ Departamento de Psiquiatria e Saúde Mental, Hospital Beatriz Ângelo and ${ }^{2}$ Departamento de Psiquiatria e Saúde Mental, Hospital Beatriz Ângelo, Instituto de Saúde Ambiental, Faculdade de Medicina, Universidade de Lisboa

${ }^{\star}$ Corresponding author.

doi: 10.1192/bjo.2021.895

Aims. The SARS-CoV-2 pandemic has led to core changes in the healthcare systems worldwide in terms of access, resources and patient's management. Patients admitted to a general hospital with COVID-19 are at a higher risk for developing or exacerbating 
various psychiatric disorders, due to the virus brain tropism and associated psychosocial factors. However, there are still limited data regarding the psychiatric needs and mental health care of these patients. The present study seeks to evaluate the changes in liaison psychiatry, patients' characteristics and outcomes, brought on by SARS-CoV-2.

Method. We retrospectively analyzed liaison psychiatry patients' characteristics during the second wave of COVID-19 pandemic, between October and December 2020, in comparison with the same trimester pre-COVID-19 in 2019.

Result. At admission to hospital, we found no differences between liaison psychiatry pre-COVID-19 $(\mathrm{n}=96)$ and COVID-19 period $(n=114)$ groups, for age $(p=0.35)$, gender $(p=0.96)$, or hospital resource utilization in the previous year : number of hospital admissions $(\mathrm{p}=.40)$, Accident and Emergency presentations ( $\mathrm{p}$ $=0.61)$, outpatients clinics $(\mathrm{p}=0.20)$. There was, however, a significant association between pre-COVID-19 vs COVID-19 period groups and psychiatric diagnosis during hospitalization $(\mathrm{X} 2(5)=$ 11.56, $\mathrm{p}=0.04)$, influencing overall respective rates of delirium/ dementias (29.2 vs $42.1 \%$ ), mood disorders (18.8 vs $26.3 \%$ ), anxiety and stress related disorders (22.9 vs $14.0 \%)$, psychoactive substance use disorders (12.5 Vs 9.6 \%) and psychotic disorders (10.4 vs $2.6 \%$ ). Mortality rate in pre-COVID-19 Vs COVID-19 period increased from $5.2 \%$ to $15.8 \%(\mathrm{X} 2(1)=5.98, \mathrm{p}=0.01)$, mostly due to increased mortality in COVID-19 patients (7.3 vs $30.3 \%, \mathrm{p}<0.001)$. Referrals to liaison psychiatry increased by $18.8 \%$ during the COVID-19 period, $28.8 \%$ of referred patients were positive for SARS-CoV-2 and time to referral was positively correlated with hospital length of stay $(\rho=0.60, p<0.001)$. In the COVID-19 period, fewer patients were referred to outpatient psychiatric follow-up after discharge from hospital $(p<0.001)$, and delayed discharges for social reasons were of shorter duration $(\mathrm{p}=0.001)$.

Conclusion. Our findings are consistent with emerging data on comorbid or superimposed mental illness in hospitalized patients with COVID-19, showing a strong impact in neuropsychiatric mani-festations, clinical prognosis and mortality, as well as clinical management. Consideration should also be given to adequate staffing of psychiatry liaison services during the pandemic, in order to attend and safely manage general hospital inpatients mental health needs.

\section{Physical health of patients under EIP - a service evaluation}

Anu Priya ${ }^{1 \star}$, Keerthan Dhanasekar ${ }^{2}$, James Hill-Cousins ${ }^{2}$, Mudasar Aziz ${ }^{2}$, Osama Suleiman ${ }^{2}$, Katherine Mills ${ }^{2}$ and Megan Lloyd ${ }^{2}$

${ }^{1}$ Bassetlaw Mental Health Services and ${ }^{2}$ Sheffield University ${ }^{*}$ Corresponding author.

doi: 10.1192/bjo.2021.897

Aims. The aim of the project was to get a baseline of the number of patients who have had blood tests, ECG and physical health observations completed in the past 12 months.

Method. There are 30 patients under Early Intervention in Psychosis team at Bassetlaw Hospital , Nottinghamshire. The elctronic notes and blood reporting system were checked for each of the patients, to get the data on blood test results , ECG reports and Physical health (Blood pressure, heart rate and weight) .

Result. It was noted that $19 / 30$ patients had Blood tests completed, 14/30 had ECG completed and 19/30 had physical health checks completed. All these patients except one were on antipsychotic medications.
Conclusion. Further work is still required in getting $100 \%$ results for all these different variables. This may include the need to review the process of how we engage the patients for physical healthcare checks. With the inclusion of a physical healthcare worker now, we might be able to improve results. Hence this evaluation would be redone in a years' time.

\section{Balint style case-based discussion group for medical students in Bassetlaw Hospital}

Anu Priya* and Hardev Bhogal

Bassetlaw Mental Health Services

${ }^{\star}$ Corresponding author.

doi: 10.1192/bjo.2021.898

Aims. To evaluate feedback from Balint style case based discussion groups and to reflect on learning points.

We have three medical students from Sheffield University on six week psychiatry placements at Bassetlaw Hospital and we get eighteen students in a year. In order to further develop their approach towards reflection and their understanding towards the doctor patient relationship we developed the Balint style case based discussion group, and each group of students attend three sessions during their placement.

Method. The groups are held on a weekly basis and consist of the three medical students and 1-2 facilitators. As the group is small one of the facilitators may participate with the students for the Balint process and to help encourage the students. Following completion of the third session of the discussion group we gain written feedback from the students. A total of 17 feedbacks have been reviewed over the period of November 2018 -November 2019.

Result. 16 students stated that this was their first experience at Balint Group and all except one student felt that they were given a good introduction about Balint groups before starting. When asked about the most significant thing that they have learnt in the group, the majority of students marked reflecting feelings to improve relationships with patients, exploring why they feel a certain way with patients and that the doctor patient relationship can affect the consultation.

One student stated that they would not recommend it to colleagues as they felt it was relevant more to doctors rather than medical students. Another student recommended having more people in a group.

Conclusion. Overall, it has been a positive experience with the medical students during the groups and with feedbacks. We have reflected on difficult topics like bereavement, fantasized about the purpose of a patient's delusion and shared the joy of a patient who was discharged after a long stay. While we think we have been able to teach the students some tips on reflection, we ourselves have been able to reflect on certain topics we would not have if not raised by the students. Some medical students have contacted the larger Balint Group in Sheffield for further sessions. Considering our experience, we will continue with the sessions at Bassetlaw Hospital.

\section{Adult ADHD clinics in north Wales - case load prevalance \& compliance with nice guidelines (quality evaluation project) \\ Jawad Raja ${ }^{1 \star}$, Zeenish Azhar ${ }^{2}$ and Alberto Salmoiraghi ${ }^{3}$ \\ ${ }^{1}$ ST6 GA Trainee Betsi Cadwaladr University Health Board; ${ }^{2}$ CT3 \\ Trainee Betsi Cadwaladr University Health Board and ${ }^{3}$ Medical \\ Director Betsi Cadwaladr University Health Board \\ ${ }^{\star}$ Corresponding author.}

doi: 10.1192/bjo.2021.899 